\title{
Fabrication of flexible gas sensor based on tin oxide by inkjet printing technology
}

\author{
O.KASSEM ${ }^{1,2 *}$, M.SAADAOUI ${ }^{2}$, M.Rieu $^{1}$, J.P.Viricelle $^{1}$ \\ ${ }^{1}$ École Nationale Supérieure des Mines, SPIN-EMSE, CNRS:UMR5307, LGF, F-42023 Saint-Étienne, \\ France \\ ${ }^{2}$ Ecole Nationale Supérieure des Mines de Saint Etienne, CMP-EMSE, F-13048 Gardanne, France
}

*Correspondence: omar.kassem@emse.fr ; Tel.: +33442616766

\begin{abstract}
In this work, a flexible tin oxide $\left(\mathrm{SnO}_{2}\right)$ gas sensor was successfully fabricated by inkjet printing technology. This thin film deposition technique requires the formulation of stable suspensions with specific fluidic properties. Sol-gel method was applied to synthesize a stable sol based on tin oxide, then transformed into ink with the appropriate rheological properties to be printed using a drop-ondemand piezoelectric inkjet printer. Thermal analysis by TGA/DSC and microstructural analysis by XRD of synthetized sol show that a crystallized structure of $\mathrm{SnO}_{2}$ could be obtained at $350{ }^{\circ} \mathrm{C}$, which is lower than crystallization temperatures of $\mathrm{SnO}_{2}$ previously reported in the literature, and entirely consistent with our plastic substrate. The printed thin-film was then sintered at $350^{\circ} \mathrm{C}$ on a flexible plastic (Upilex-50S).
\end{abstract}

Key words: Flexible gas sensor, $\mathrm{SnO}_{2}$ ink preparation, Inkjet Printing

\section{Introduction}

In recent years, a significant advance in the development and implementation of flexible sensors has demonstrated the increasing utility of these special type of sensing platforms [1]. In particular, flexible gas sensors based on metal oxide belong to this category, and have an important role in environmental applications. Tin oxide is considered to be one of the most useful materials in gas sensing applications because of its remarkable sensing properties, high chemical stability, and ease of integration during sensor design. The manufacture of flexible gas sensors based on tin oxide thin film is subject to restrictions as it requires a high operating temperature which is not compatible with all plastic foils. In our present work, inkjet printing was adopted as the deposition technique in order to print flexible tin oxide gas sensing films in additive way. A stable sol was synthetized by sol-gel method, and used as a precursor ink with appropriate rheological properties. The electrical response of the sensor to $\mathrm{CO}$ gas was characterized.

\section{Experimental part}

The first step consists in synthesizing a stable sol from a precursor based on a tin salt by aqueous sol-gel route using acetic acid and ethylene glycol as complexing ligands. Thermal analyses of the obtained sol were carried out by thermo-gravimetric analysis (TGA) and differential scanning calorimetry (DSC), from $25^{\circ} \mathrm{C}$ to $800{ }^{\circ} \mathrm{C}$ in order to investigate the evaporation of solvents and crystallization of $\mathrm{SnO}_{2}$.

Once the sol is synthesized, it is transformed into ink with an appropriate rheology to satisfy the printability criteria of the Dimatix printer (DMP-2800 Fujifilm). Viscosity and surface tension were tuned by adding certain proportions of solvents and additives. To prepare sensor electrodes, gold was deposited on Upilex by evaporation under vacuum, and then a $1064 \mathrm{~nm}$ Laser beam was used to pattern gold electrodes onto foil with an interspace of $200 \mu \mathrm{m}$. $\mathrm{SnO}_{2}$ ink was printed onto these electrodes using commercial Dimatix printer (DMP-2800 Fujifilm), and heated at $350^{\circ} \mathrm{C}$ for 1 hour in oven. Electrical characterization of the fabricated sensor was 
performed using different concentrations of $\mathrm{CO}$ gas, at $300^{\circ} \mathrm{C}$ in dry air.

\section{Results and Discussion}

Figure 1 shows weight loss and heat flow of the sol as a function of temperature, as obtained from TGA/DSC. A weight loss between 100 and $210^{\circ} \mathrm{C}$ is clearly observed which is related to the removal of acetic acid and ethylene glycol. These two evaporation phases are associated with two endothermic peaks as observed in the DSC curve. The first peak at $90^{\circ} \mathrm{C}$ corresponds to the evaporation of the weakly bounded acetate groups and the second one at $200^{\circ} \mathrm{C}$ corresponds to the evaporation of ethoxy bounds [2]. Finally, an exothermic peak at $350^{\circ} \mathrm{C}$ observed on DSC curve is associated with the formation of stable $\mathrm{SnO}_{2}$. This crystallization temperature is well suited for plastic foil.

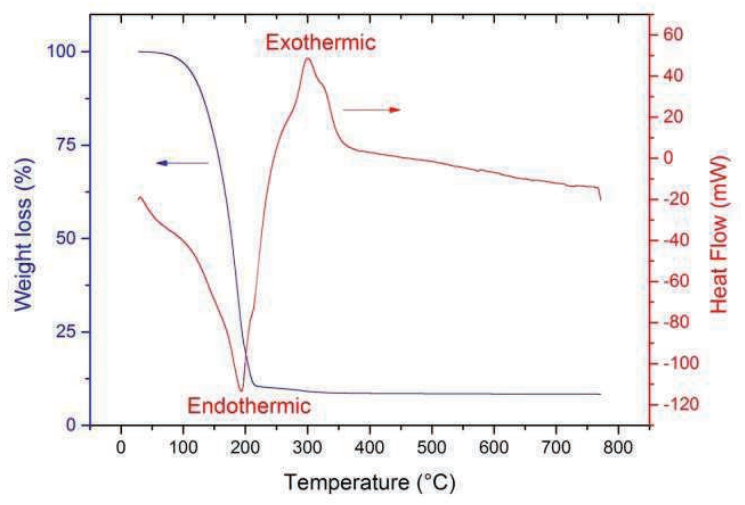

Figure 1: TGA/DSC thermograms of the $\mathrm{SnO}_{2}$ sol

Once the sol is synthesized, it is transformed into ink with an appropriate $\mathrm{pH}$, viscosity and surface tension. A combination of three solvents as ethanol, ethylene glycol and glycerin was used to adjust the viscosity to $10 \mathrm{cP}$ and the surface tension to $32 \mathrm{mN} / \mathrm{m}$. The adjustment of ink rheology in regards to solvent can be characterized by dimensionless $Z$ number, which is the inverse of the Ohnesorge $(\mathrm{O} h)$ number defined as:

$$
O h=\frac{\eta}{\sqrt{\gamma \cdot \rho \cdot a}}
$$

where $\rho, \eta$, and $y$ are respectively the density, dynamic viscosity, and surface tension of the fluid and "a" is a characteristic length of the cartridge nozzle [3]. $Z$ must be between 1 and 10 for stable drop formation

A $\mathrm{SnO}_{2}$ ink with a $\mathrm{Z}$ number of (2.7) was successfully printed onto Upilex between two gold electrodes and sintered at $350^{\circ} \mathrm{C}$ for $1 \mathrm{~h}$.

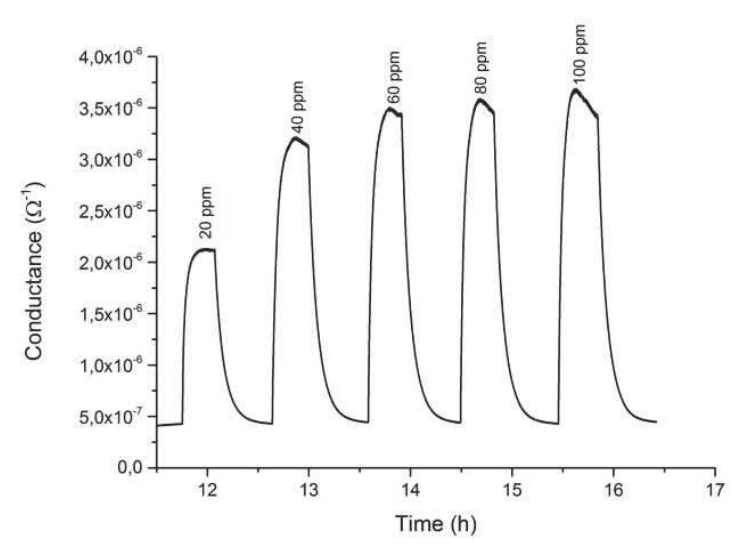

Figure 2: The change of conductance of $\mathrm{SnO}_{2}$ thin film, upon exposure to different concentrations of $\mathrm{CO}$ gas in dry air at $3000^{\circ}$.

Figure 2 shows the response of $\mathrm{SnO}_{2}$ flexible sensor at $300{ }^{\circ}$. The conductance increases upon exposure to different concentration of $\mathrm{CO}$ gas (20-100ppm), and it drops rapidly and returned to its original state when the injection of $\mathrm{CO}$ gas is stopped in the testing atmosphere in each cycle, indicating that the gas sensor has a fast and reversible response for different $\mathrm{CO}$ concentrations.

\section{Conclusion}

A flexible $\mathrm{SnO}_{2}$ gas sensor was successfully prepared by inkjet printing. $\mathrm{SnO}_{2}$ precursor solution was synthetized using aqueous sol-gel method. Thermal analysis by TGA/DSC has shown that a crystallized structure of $\mathrm{SnO}_{2}$ could be obtained at $350^{\circ} \mathrm{C}$, which is entirely consistent with our plastic substrate. Electrical characterizations have demonstrated that the deposited layer by inkjet has adequate properties for gas sensing. We are working now on printing a metallic heater and electrodes to manufacture a fully inkjet flexible gas sensor.

\section{References}

[1] Kenry, J. C. Yeo, and C. T. Lim, "Emerging flexible and wearable physical sensing platforms for healthcare and biomedical applications," Microsyst. Nanoeng., vol. 2, no. 1, Dec. 2016.

[2] H. Köse, Ş. Karaal, A. O. Aydin, and H. Akbulut, "Structural properties of size-controlled $\mathrm{SnO} 2$ nanopowders produced by sol-gel method," Mater. Sci. Semicond. Process., vol. 38, pp. 404-412, Oct. 2015.

[3] B. Derby, "Inkjet Printing of Functional and Structural Materials: Fluid Property Requirements, Feature Stability, and Resolution," Annu. Rev. Mater. Res., vol. 40, no. 1, pp. 395-414, Jun. 2010. 